\title{
ASYMMETRIC DROP MARANGONI MIGRATION OF LARGER REYNOLDS NUMBERS $\dagger$
}

\author{
R. H. GENG and W. R. HU \\ Institute of Mechanics, CAS, Beijing, 100080, People's Republic of China \\ C. $\mathrm{AO}$ \\ Institute of China Computational Mathematics, CAS, Beijing, 100080, People's Regpublic of China
}

(Received 25 April 1997)

\begin{abstract}
Two-dimensional and unsteady problems of drop and bubble Marangoni migration are calculated for cases of larger Reynolds numbers in the microgravity environment. A global treatment method is introduced to solve numerically the complete problem by using the finite differential method, and the thermocapillary force acting on the interface is considered as a body force acting in the computational mesh elements which are passed by the interface. The program of numerical simulation is checked with the symmetric model of bubble migration in Ref. [1]. The asymmetric model of bubble migration is calculated, and the results are different from that of the symmetric model. The asymmetric model of drop migration is also calculated. The trajectory of drop in larger Reynolds number fluid is oscillated periodically due to the vortex separation in the wake. (C) 1998 Elsevier Science Ltd. All rights reserved
\end{abstract}

\section{INTRODUCTION}

The Marangoni migration of drop and bubble, coupled with the effect of gravity, has been studied extensively since the pioneer work of Young, Goldstein and Block, and named as the YGB model[1]. The Maragoni migration becomes more important in the microgravity science, because interface phenomena often dominate the microgravity process and need to be studied in order to understand the mechanism and its many applications.

The YGB model analytically deals with the linear case of small Reynolds number $(\mathrm{Re} \ll 1)$ and small Marangoni numbers $(\mathrm{Ma} \ll 1)$ by using the perturbation method. The analytical solutions for bubble [2] and drop [3] were obtained to include the influence of inertia term $(\operatorname{Re}>1)$ but omitting the terms of heat convection $(\mathrm{Ma} \ll 1)$, or to include the influence of heat convection $(\mathrm{Ma} \gg 1)$ but omitting the inertia term $(\operatorname{Re} \ll 1)[4-6]$. However, the general problem of arbitrary Reynolds numbers and Marangoni numbers should be solved by the numerical simulation, for example the bubble migration in ref. [7].

Some experiments of drop migration were performed in the microgravity. The experimental results agree with the YGB model if the Reynolds number is small [8], but the migration in microgravity environment is slower than the one of YGB model for

$\dagger$ Paper IAF-96-J3.03 presented at the 7th International Astronautical Congress, Beijing, China, 7-11 October 1996. the case of larger Reynolds numbers [9]. The ground experiments for larger Reynolds numbers gave the similar conclusion [10]. To explain the experiments of larger Reynolds numbers, the numerical simulation will be beneficial to study the process of migration, and is studied in the present paper.

The mathematical model and the calculated method are given in the next section, and then the calculated method and its correction are discussed in Section 3. The asymmetric models of Marangoni migration for larger Reynolds numbers will be analyzed, respectively, for bubble, in Section 4 and for drop, in Section 5. The last section is the conclusion.

\section{MODEL OF MARANGONI MIGRATION}

The problem of drop migration in the microgravity environment is usually described by the incompressible fluid dynamic equations with discontinuous conservation conditions at the interface. In the present paper, two-dimensional and unsteady problems are analyzed by the numerical simulation method. A Cartesian coordinate system with the $y$ axis opposite the direction of applied temperature gradient, and origin at the center of the static drop or bubble, is adopted as shown in Fig. 1. The temperature at the lower boundary is $T_{0}$. The temperature gradient, $A$, is negative and the rate of change of the surface tension with temperature, $\sigma_{\mathrm{T}}$, is also negative. The drop moves from the cold regions toward the hotter regions. The coordinate system is fixed in the laboratory but does not move together with the drop or bubble. In this case, the basic equations may be 
written as follows

$$
u_{x}+v_{y}=0
$$

$u_{t}+u u_{x}+v u_{y}=-\rho_{x} / \rho+\left(2 v u_{x}\right)_{x}+\left[v\left(u_{y}+v_{x}\right)\right]_{y}+f_{x}$,

$$
\begin{aligned}
v_{t}+u v_{x}+v v_{y}= & -p_{y} \rho+2\left(v V_{Y}\right)_{Y} \\
& +\left[v\left(U_{Y}+V_{X}\right)_{X}+F_{Y},\right. \\
T_{t}+u T_{x}+v T_{y}= & \left(T_{x} \kappa\right)_{x}+\left(T_{y} \kappa\right)_{y}+v\left[2 u_{x}^{2}\right. \\
& \left.+2 v_{y}^{2}+\left(u_{y}+v_{x}\right)^{2}\right] / c_{p},
\end{aligned}
$$

where $u, v, t, p, \rho, c_{\mathrm{p}}, \kappa, v$, are, respectively, the velocity components in the $x$ - and $y$-direction, temperature, pressure, density, specific heat capacity, thermal conductivity, and kinetic viscosity, $\mathrm{f}_{x}, \mathrm{f}_{y}$ are respectively components of the body force. Subscripts $t, x$ and $y$ denote respectively the partial differential to $t, x$ and $y$. In the present paper, the thermocapillary force acting on the interface is considered as a body force acting in computational mesh elements which are passed by the interface. In this case, there are no special boundary conditions at the interface. The associated boundary conditions of the problem are given as follows:

$$
T=T_{0}+A\left(y-y_{0}\right),
$$

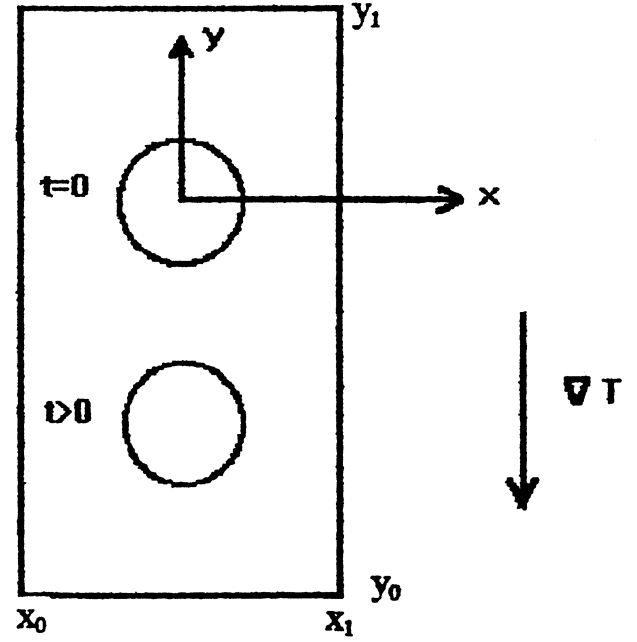

Fig. 1. Cartesian coordinate system of the model.

$$
u=0, \quad v_{x}=0,
$$

at the left $\left(x=x_{0}\right)$ and right $\left(x=x_{1}\right)$ boundaries; and

$$
\begin{gathered}
v=0, \quad u_{y}=0, \\
T=T_{0}, \quad\left(y=y_{0}\right) \\
T=T_{0}+A\left(y_{1}-y_{0}\right), \quad\left(y=y_{1}\right)
\end{gathered}
$$

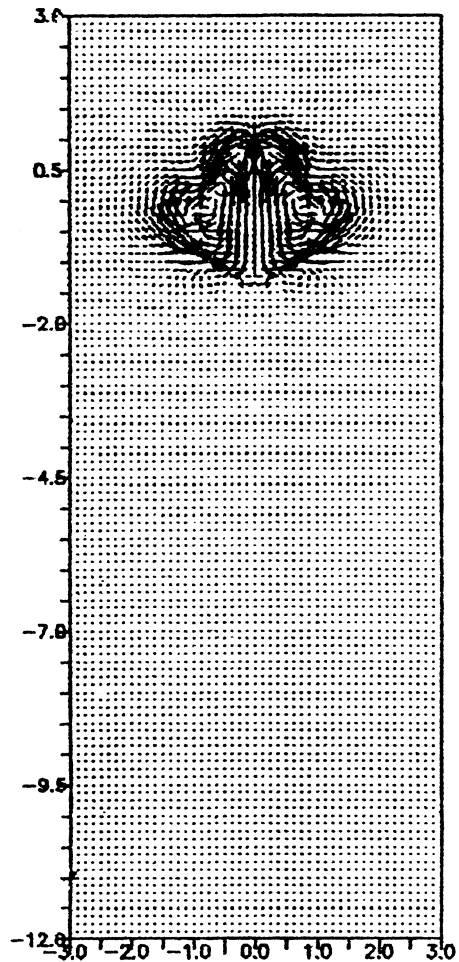

(a) $\mathrm{t}=1 \mathrm{~s}$

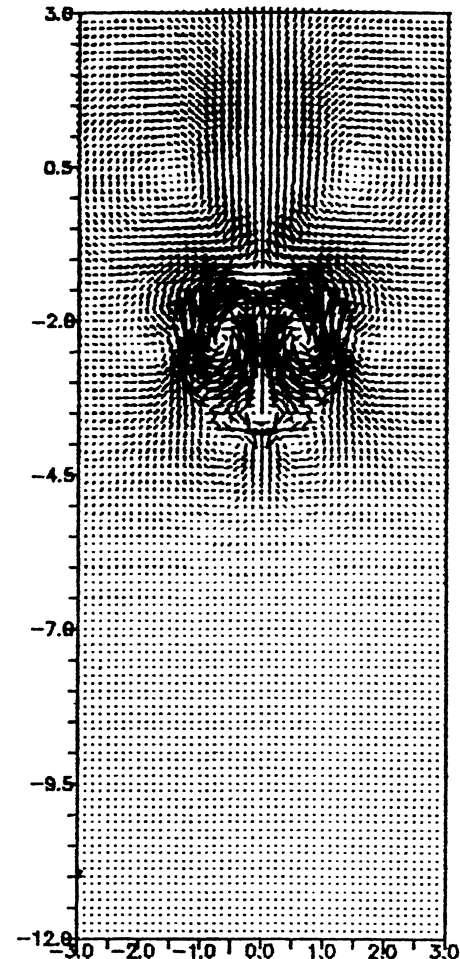

(b) $\mathrm{t}=10 \mathrm{~s}$

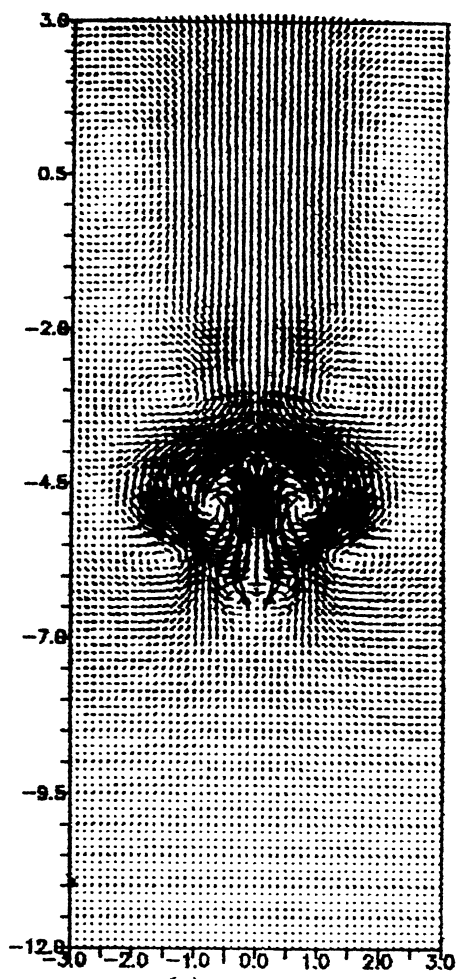

(c) $t=26 \mathrm{~s}$

Fig. 2. The bubble velocity vector fields under the symmetric assumption. 

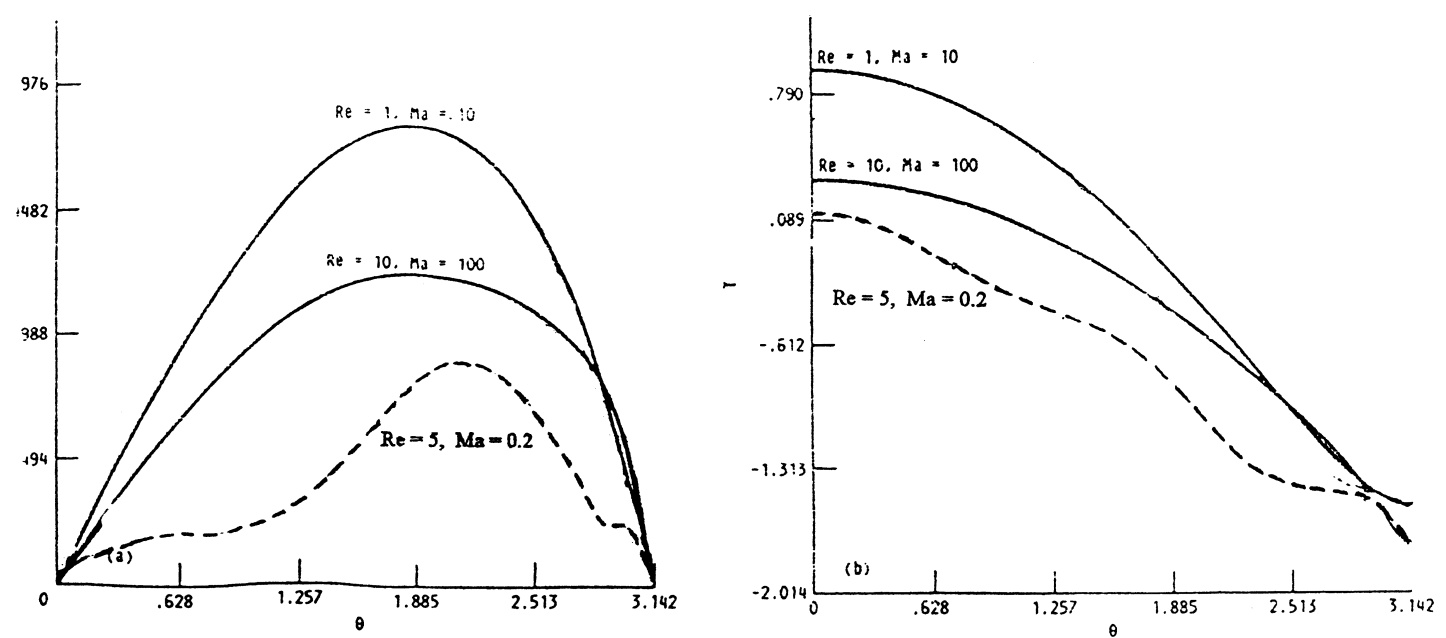

Fig. 3. (a). Tangential velocity distribution on the bubble surface. (b). Temperature distribution on the bubble surface. $(----)$ for this present, (- $(-)$ for Ref. [1].

at the upper $\left(y=y_{0}\right)$ and $\left(y=y_{1}\right)$ lower boundaries, where $A$ and $T_{0}$ are, respectively, constants of applied temperature gradient and temperature at the lower boundary. $x_{0}, x_{1}$ and, $y_{0}, y_{1}$ are, respectively boundary coordinates value of calculating domain as shown in Fig. 1.
The initial conditions are given as:

$u=0, \quad v=0, \quad T=T_{0}+A\left(y-y_{0}\right) . \quad$ at $t=0$.

Then, the problem is described by eqns (1)-(4), boundary conditions (5)-(9), and initial conditions

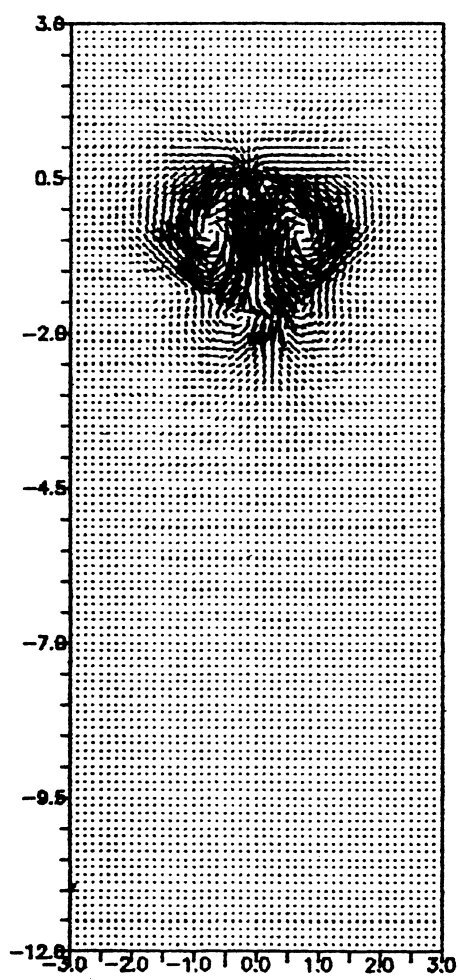

(a)

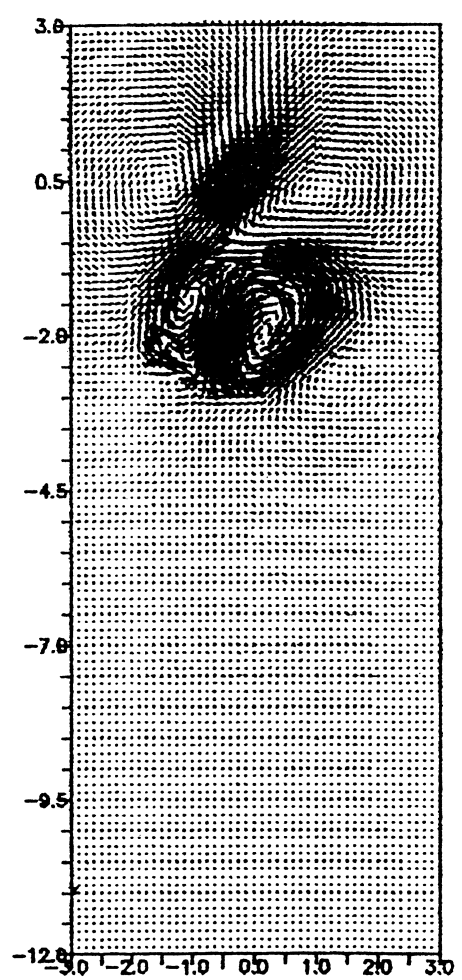

(b)

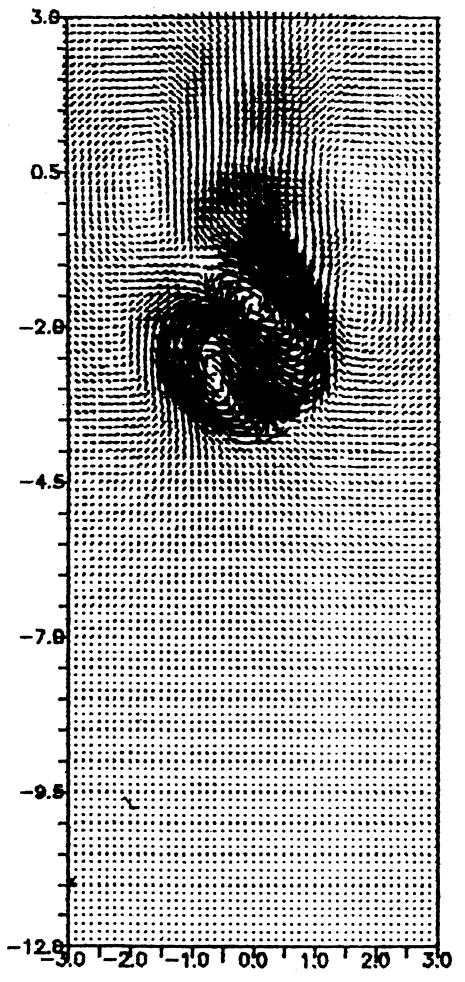

(c)

Fig. 4. The bubble velocity vector fields without the symmetric assumption; (a) $t=2 \mathrm{~s}$. The center of bubble is $(0.012,-0.602)$; (b) $t=6 \mathrm{~s}$. The center of bubble is $(-0.449,-1.818)$; (c) $t=8.6 \mathrm{~s}$. The center of bubble is $(0.043,-2.651)$. 


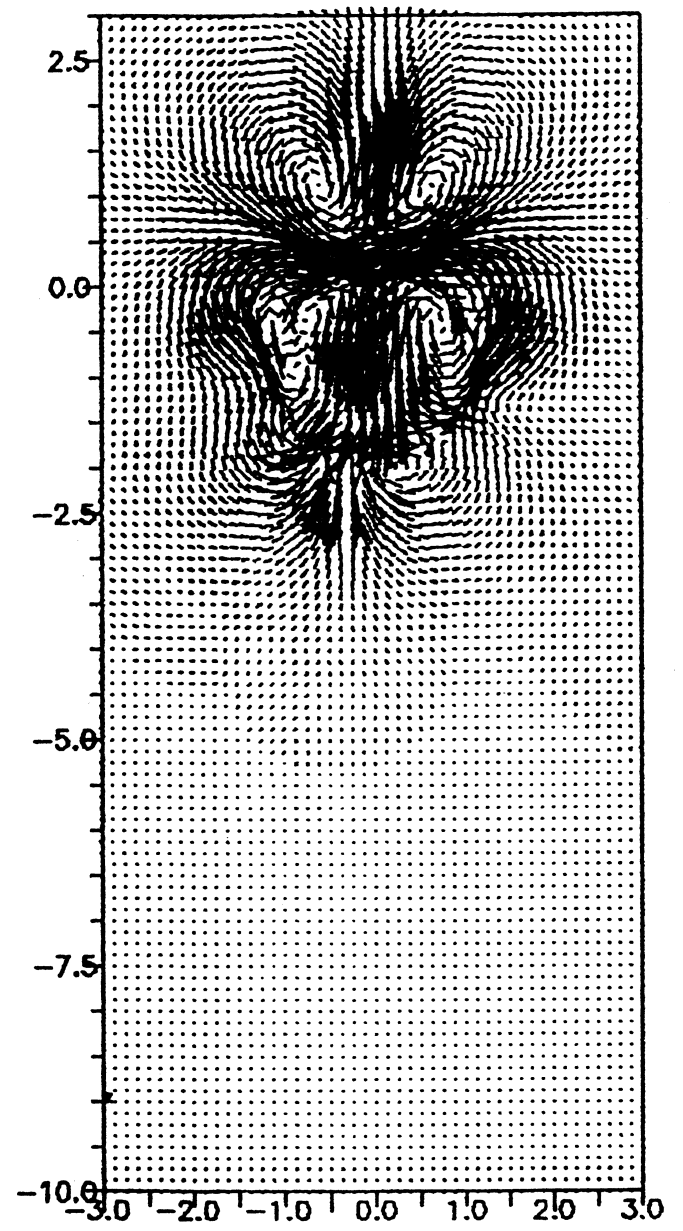

(a)

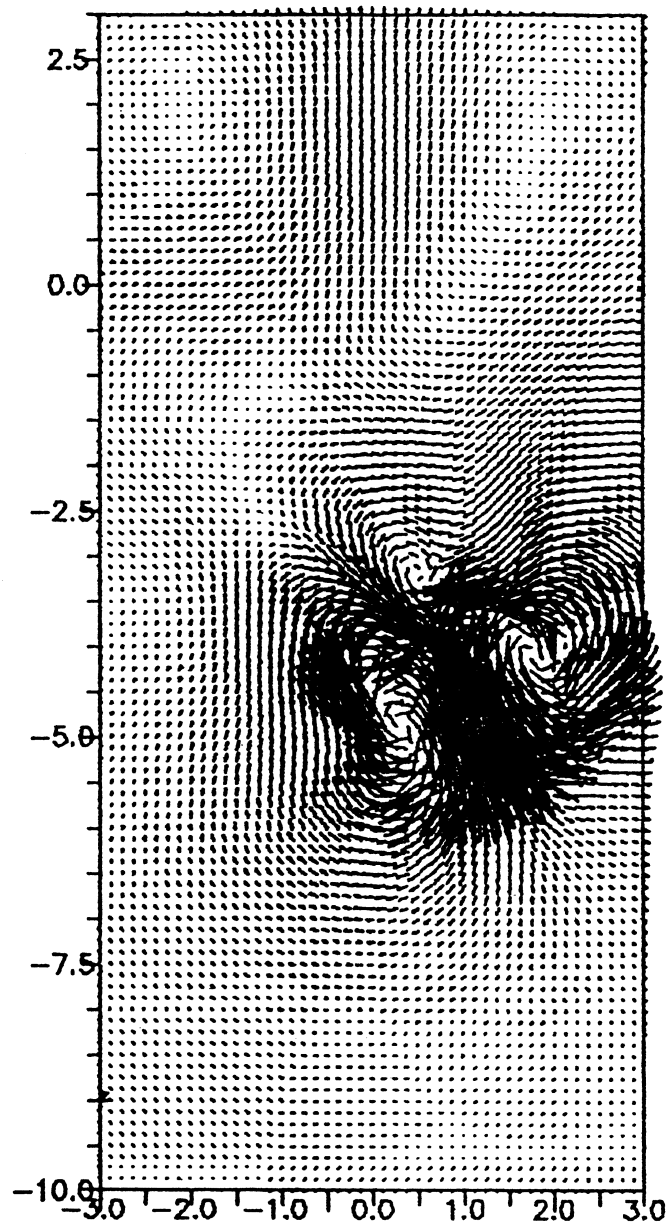

(b)

Fig. 5. The drop velocity vector fields; (a) $t=4.5 \mathrm{~s}$, the center of drop is $(-0.036,-1.124)$, (b) $t=15 \mathrm{~s}$, the center of drop is $(1.243,-4.909)$.

(10), and was solved by the following numerical method.

\section{COMPUTATIONAL METHOD}

The basic eqns (1)-(4) were solved in a $2 \mathrm{D}$ rectangular domain $\left(x_{0} \leq x \leq x_{1}, y_{0} \leq y \leq y_{1}\right)$ by a finite difference method of staggered grid [11]. The geometric constants $x_{0}, x_{1}, y_{0}, y_{1}$ are adopted respectively as $-14,14,-28,10$, and the diameter of bubble or drop is two. The width of computational grid of the drop is adopted as 1/32 diameter.

Following the iterative method suggested in ref. [11], the equations were solved with the pressure unchanged, and then both the pressure and the velocities were modified, by the pressure and velocity correction method, to obtain new values of these quantities for the next step. In the interfacial regions, the physical properties, such as constants $\rho, c_{p}, \kappa$, and $v$, should be discontinuous across the interface in physics. During the present calculation, a smooth distribution with sharp variation is intro- duced for approximation of the discontinuous distribution, such as $g=g_{0}+\left(g_{1}-g_{0}\right) \operatorname{SI}(x, y)$, where $g_{0}, g_{1}$ are, respectively, the outer and inner value of the function $g\left(g=\rho, c_{p}, \kappa, v\right)$, and

$$
\operatorname{Sl}(x, y)= \begin{cases}0 & \text { outside the drop } \\ V_{1} / V_{0} & \text { near the interface } \\ 1 & \text { inside the drop }\end{cases}
$$

where $V_{1}$ and $V_{0}$ are respectively the volume of the drop and outer fluid in the grid. This approach successfully overcomes the difficulties of discontinuities of physical properties across the interface[12]. In this paper, the time step is adopted by condition $\Delta t<(\Delta y / 40) / v_{\max }$, where $\Delta y$ and $v_{\max }$ are, respectively, the width in the $y$-direction of a calculating grid, and the maximum of velocity components. The Reynolds number is defined as $\operatorname{Re}=-\sigma_{T} \mathrm{~A} R^{2} /$ $\mu \nu$, and the Prandtl number $\operatorname{Pr}=v / \kappa$. All the parameters are given for bubble or drop.

Supposing that $\left(x_{i}(l), y_{i}(l)\right)$ are the points in interface $(l=1,2, \ldots)$, and $T_{i}(l), \gamma_{0}$ are, respectively, 


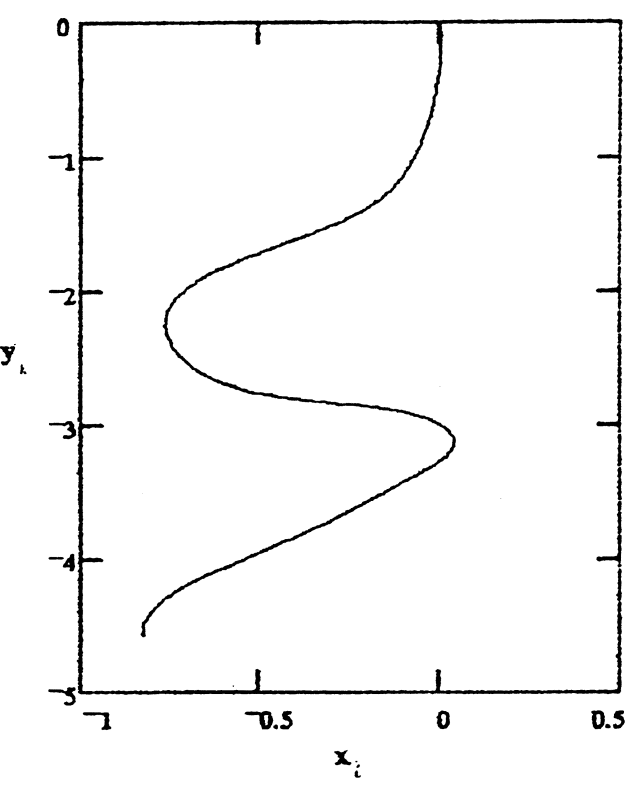

(a)

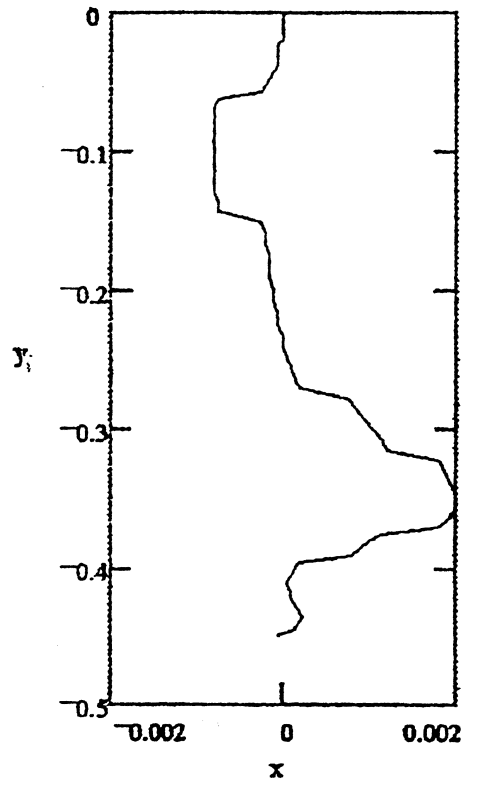

(b)

Fig. 6. The trajectory of (a) bubble, (b) drop without the symmetric assumption.

temperatures and surface tension at these points. $L(l) \quad$ are segments from $\left(x_{i}(l), y_{i}(l)\right)$ to $\left(x_{i}(l+1), y_{i}(l+1)\right)$ and $\left(x_{m}(l), y_{m}(l)\right)$ are middle points of segments $L(l), R(l)$ are radial curvatures of points $\left(x_{m}(l), y_{m}(l)\right)$. Then the body force of normal component $\left(f_{x}^{n}(l), f_{y}^{n}(l)\right)$ and tangential component $\left(f_{x}^{\tau}(l), f_{y}^{\tau}(l)\right)$ due to the surface tension at points $\left(x_{m}(l), y_{m}(l)\right)$ are calculated as follows:

$$
\begin{aligned}
& f_{x}^{n}(l)=\gamma_{0}\left[y_{i}(l+1)-y_{i}(l)\right] / R(l), \\
& f_{y}^{n}(l)=\gamma_{0}\left[-x_{i}(l+1)+x_{i}(l)\right] / R(l),
\end{aligned}
$$

$$
\begin{aligned}
& f_{x}^{\tau}(l)=-\sigma_{T}\left[T_{i}(l+1)-T_{i}(l)\right]\left[x_{i}(l+1)-x_{i}(l)\right], \\
& f_{y}^{\tau}(l)=-\sigma_{T}\left[T_{i}(l+1)-T_{i}(l)\right]\left[y_{i}(l+1)-y_{i}(l)\right] .
\end{aligned}
$$

The body force in Cartesian coordinate system is then given as:

$$
\begin{aligned}
& f_{x}(l)=f_{x}^{n}(l)+f_{x}^{\tau}(l), \\
& f_{y}(l)=f_{y}^{n}(l)+f_{y}^{\tau}(l) .
\end{aligned}
$$

All the body force $f_{x}(l), f_{y}(l)$ are given in their adjacent mesh points according to their weights.

The computational method is checked and compare with the bubble migration of the symmetric model given in ref. [7], and the assumption of nondeformation drop is also used. Figure 2 are the velocity vector fields at different times under the symmetric assumption.

Figure 3 show the comparison of velocity and temperature at the bubble surface given by ref. [7] and this paper for the symmetric model. The basic trends of temperatrue and tangential velocity distribution on the bubble surface are similar, although the Reynolds and Prandtl numbers are different, and the coordinate system of the spherical model in ref. [7] and the cylindrical model in the present paper are different.

\section{BUBBLE MARANGONI MIGRATION}

The bubble Marangoni migration of the asymmetric model was considered for $\mathrm{Re}=5$ and $\operatorname{Pr}=0.04$. The bubble migrates from the initial position toward the hot side of the enclosure.

Fig. 4 are the flow fields for the asymmetric model during the migration, and the trajectory of the bubble center can be seen in Fig. 6(a), where the bubble staggers all the time.

There are some differences between the symmetric and asymmetric migrations of the bubble. Inside the bubble, the two vortex cells in Fig.2. are symmetric about the vertical line of the domain, but these in Fig.4, are symmetric about a line along the moving direction due to the asymmetric wake.

\section{ASYMMETRIC DROP MIGRATION}

The drop Marangoni migration is investigated in this section. Calculations were performed for $R e=2.6$ and $\operatorname{Pr}=105$. The velocity fields of the asymmetric model during the migration are shown in Fig. 5. The drop also moves from the colder regions toward the hotter regions. The drop staggers along the vertical line due to the asymmetric distribution of the pressure in wake. Its trajectory, as shown in Fig.6.(b), is similar to the asymmetric model of bubble and the two vortex cells inside the 
drop are not symmetric about the vertical line of the domain but about a line along the moving direction.

\section{CONCLUSION}

A new method of numerical simulation was applied to calculate the problem of Marangoni migration, the basic equations are satisfied in the whole calculation region and the surface force is replaced by a body force acting in the small mesh region. In this case, the interface does not need to be considered as the discontinuity surface to separate two different kinds of liquid. In fact, usual method of numerical simulation treats the interface as a discontinuous surface, however, the discontinuous conditions at the interface should also be satisfied in the small region of calculated mesh, and the solution is often obtained by many iterations. The results of the present paper show that this new method is successful in solving the problem with discontinuous interfaces such as the drop and bubble migration.

In the present paper, the drop and bubble Marangoni migration of larger Reynolds number fluid were studied for the asymmetric model, and the asymmetric features are much more complex than that of the linear model. Non-linear features obviously apear in the wake with vortex separation, and then the trajectory of the drop or bubble migration is oscillatory around the vertical line of the domain (Fig.6.). Because, in our simulation, the site of the interface is only a mark, maybe it is the reason that the central line is not, but parallel to the symmetric axis; this effect will be studied later.

For simplification, there are some assumptions in the present paper, such as the $2 \mathrm{D}$ model and nondeformation of the interface. Three-dimensional and the deformation interface model in different parameter ranges should be studied in the future.

\section{REFERENCES}

1. Young, N. O., Goldstein, J. S. and Block, M. J., The motion of bubbles in a vertical temperature gradient, J. Fluid Mechanics, 1959, 6, 350.

2. Balasubramaniam, R. and Chai, An-Ti. Thermocapillary migration of droplets: an exact solution for small Marangoni numbers, J. Colloid Interface Science, 1987, 119, 531.

3. Haj-Hariri, H., Nadim, A. and Borhan, A., Effects of inertia on the thermocapillary velocity of a drop, $J$. Colloid Interface Science, 1990, 140, 277.

4. Sabramanian, R. S., AIChE Journal, 1981, 27, 646.

5. Shanker, N. and Subramanian, R. S., The Stokes motion of a gas bubble due to interfacial tension gradients at low to moderate Marangoni numbers, $J$. Colloid and Interface Science, 1988, 123, 512.

6. Merritt, R. M., Mortan, D. S. and Subramanian, R. S., Flow structures in bubble migration under the combined action of boyancy and thermocapillarity, $J$. Colloid Interface Science, 1993, 155, 200.

7. Balasubramaniam, R. and Lavery, J. E., Numerical simulation of thermocapillary bubble migration under microgravity for large Reynolds and Marangoni numbers, Numerical Heat Transfer, 1989, 16, 175-187.

8. Braum, B., Ikier, C. and Klein, H., Thermocapillary migration of droplets in a binary mixture with miscibility gap during liquid/liquid phase separation under reduced gravity, J. Colloid Interface Science, 1993, 159, 515.

9. Wozuiak, G., The thermocapillary motion of droplets under reduced gravity, J. Colloid Interface Science, 1991, 141, 245.

10. Xie, J. C., Lin, H., Han, J. H. and Hu, W. R., Drop migration of middle Reynolds number in a verticle temperature gradient, Microgravity Science and Tech, (in press).

11. Huang, L. C. and $\mathrm{Wu}, \mathrm{Ya}$-dan. Implicit projection method for the incompressible N-S equations.

12. Unverdi, S. O. and Tryggvason, G., A front-tracking method for viscous, in compressible, multifluid flows, J. Computational Physics, 1992, 100, 25-37. 